\title{
Asymptotic Behaviors for Delay Lotka-Volterra Model Disturbed by G-Brownian Motion
}

\author{
Ping He $\left.\mathbb{D}^{1}\right)^{1,2}$ Yong Ren ${ }^{(D)},{ }^{1}$ and Defei Zhang $\mathbb{D}^{2}$ \\ ${ }^{1}$ Department of Mathematics, Anhui Normal University, Wuhu 241000, China \\ ${ }^{2}$ Department of Mathematics, Honghe University, Mengzi 661199, China
}

Correspondence should be addressed to Defei Zhang; zhdefei@163.com

Received 30 December 2019; Accepted 10 February 2020; Published 7 April 2020

Guest Editor: Wenguang Yu

Copyright $\odot 2020$ Ping He et al. This is an open access article distributed under the Creative Commons Attribution License, which permits unrestricted use, distribution, and reproduction in any medium, provided the original work is properly cited.

In this paper, we propose the stochastic Lotka-Volterra model with delay disturbed by G-Brownian motion $\mathrm{d} x=\operatorname{diag}\left(x_{1}, x_{2}, \ldots, x_{n}\right)[(A x(t-\tau)+b) d\langle B\rangle(t)+\sigma x \mathrm{~d} B(t)]$. Under a natural assumption on noise, we study existence and uniqueness of the global positive solution for the system and its asymptotic pathwise moment behavior and prove that the solution does not explode to infinity in a finite time.

\section{Introduction}

Since the Lotka-Volterra model (LVM in short) was provided by Lotka [1] and Volterra [2], there were extensive works concerned with the dynamics of this system and global stability and the stochastic Lotka-Volterra population model, and in here, we only mention $[3,4]$ (for deterministic situation) and [5-8] (for stochastic situation). The wellknown two-dimensional delay Lotka-Volterra ecological population model driven by Brownian motion is

$$
\left\{\begin{array}{l}
\mathrm{d} x_{1}=x_{1}\left[\left(a_{1}+b_{11} x_{1}(t-\tau)+b_{12} x_{2}(t-\tau)\right) \mathrm{d} t\right. \\
\left.+\left(c_{11} x_{1}+c_{12} x_{2}\right) \mathrm{d} W(t)\right] \\
\mathrm{d} x_{2}=x_{2}\left[\left(a_{2}+b_{21} x_{1}(t-\tau)+b_{22} x_{2}(t-\tau)\right) \mathrm{d} t\right. \\
\left.+\left(c_{21} x_{1}+c_{22} x_{2}\right) \mathrm{d} W(t)\right] .
\end{array}\right.
$$

Bahar and Mao in [9] proved that the solution of (1) is almost surely nonnegative and finite. $\mathrm{Wu}$ and $\mathrm{Xu}$ in [10] investigated stochastic LVM with infinite delay. Global asymptotic stability for a stochastic delay LVM was obtained in [11].

Peng first established the stochastic analysis theory under the $G$-expectation framework in references [12-14]. Peng's G-expectation space is an essential extension for probability measure space. Since then, many important theoretical results in this field are obtained, for example, SLL for sublinear expectations are obtained in [15], capacity theory results are discussed in [16] and [17-19], and other related technologies in [20-22]. Inspired by these results, we investigate a stochastic delay Lotka-Volterra model disturbed by $G$-Brownian motion:

$$
\mathrm{d} x=\operatorname{diag}\left(x_{1}, x_{2}, \ldots, x_{n}\right)[(b+A x(t-\tau)) \mathrm{d}\langle B\rangle(t)+\sigma x \mathrm{~d} B(t)],
$$

with $\{x(s):-\tau \leq s \leq 0\} \in C\left([-\tau, 0] ; R_{+}^{n}\right)$, where $x=\left(x_{1}\right.$, $\left.\ldots, x_{n}\right)^{T}$ is a n-dimensional vector, $x_{i}(t)$ is the population size of species $i$ at time $t(t \geq 0), b=\left(b_{1}, b_{2}, \ldots, b_{n}\right)^{T}, b_{i}$ is the species $i$ 's growth rate, $A=\left(a_{i j}\right)_{n \times n}$ is a $n \times n$ community matrix, $a_{i j}(I \neq j)$ is the interspecific interaction effect, and $a_{i i}$ is the intraspecific interaction effect. We assume that the interaction effect in this system was disturbed by a $G$ Brownian motion with $\widehat{\mathbb{E}}\left[B(t)^{2}\right]=\bar{\sigma}^{2} t$ and $\widehat{\mathbb{E}}\left[-B(t)^{2}\right]=-\underline{\sigma}^{2} t$, where $\sigma=\left(\sigma_{i j}\right)_{n \times n}$ is a constant matrix, representing the total interference intensity matrix for the system; $B(t)$ has a variance-uncertainty but not mean-uncertainty; $\langle B\rangle(t)$ has a mean-uncertainty property. Therefore, $(\langle B\rangle, B)$ is used to characterise the disturbed growth rate, disturbed interspecific, or intraspecific interactions and interference intensity at the same time. We think the model (2) considers the stochastic interference from both 
mean-uncertainty and variance-uncertainty, but the traditional stochastic model cannot describe this property. Indeed, we prove the solution of (2) is quasi-surely nonnegative and finite. Some asymptotic pathwise moment estimations for the solutions of this system are presented.

\section{Stochastic Delay Lotka-Volterra Model Driven by $G$-Brownian Motion}

Definitions about sublinear expectations, $G$-Brownian motions, and quadratic variation process $\langle B\rangle(t)$ and notations, as well as more details can also be found in [12-14]. For a matrix $A$, we denote $|A|=\sqrt{\left(A^{T} A\right)}$ and $\|A\|=\sup \{|A x|:|x|=1\} . C\left([-\tau, 0] ; R_{+}^{n}\right)$ denotes the family of continuous functions from $[-\tau, 0]$ to $R_{+}^{n}$. We assume the matrix $\sigma$ satisfies the following assumption:

$$
\text { (A) } \begin{cases}\sigma_{i i}>0, & i \in[1, n], \\ \sigma_{i j} \geq 0, & i \neq j \in[1, n] .\end{cases}
$$

The assumption (A) was first assumed by Mao et al. in [5], and it is also necessary in our framework.
Theorem 1. If the matrix $\sigma$ in system (2) satisfies assumption (A), then $\forall A \in R^{n \times n}, b \in R^{n}$ and $\{x(s): s \in[-\tau, 0]\}$, then there exists a unique solution $x$ of equation (2). Furthermore, $x(s) \in R_{+}^{n} \quad$ for all $s \geq-\tau$ quasi-surely, namely, $v\left(\omega: x(s) \in R_{+}^{n}, s \in[-\tau, \infty)\right)=1$.

Proof. Because the coefficients of equation (2) are locally Lipschitz continuous, there exist a unique local solution $x(s)$ on $s \in\left[-\tau, \tau_{e}\right)$, where $\tau_{e}$ is called explosion time. To see it is also global, we must show $\tau_{\infty}=\infty$ q.s. Suppose $k_{0}\left(k_{0}>0\right)$ is large enough s.t. $x(t)(t \in[-\tau, 0])$ satisfies $1 / k_{0}<\min |x(t)|$, $\max |x(t)|<k_{0}$. For any $k\left(k \geq k_{0}\right)$, set $\tau_{k}=\inf \left\{s \in\left[0, \tau_{e}\right)\right.$ : $\left.x_{i}(s) \notin(1 / k, k), 1<i \leq n\right\}$, where inf $\varnothing=\infty$. Noting that $\tau_{k}$ is increasing when $k \longrightarrow \infty$, let $\tau_{\infty}=\lim _{k \longrightarrow \infty} \tau_{k}$, then $\tau_{\infty} \leq \tau_{e}$ q.s. If we can prove $\tau_{\infty}=\infty$ q.s., then $\tau_{e}=\infty$ q.s. and $x(t) \in R_{n}^{+}$q.s., $t \geq 0$. If $\tau_{\infty} \neq \infty$ q.s., then $\exists$ a constant $T>0$ s.t. $V\left(\omega: \tau_{\infty}(\omega) \leq T\right) \geq \varepsilon$ for any $\varepsilon>0$, namely, $\exists$ an integer $k_{1}\left(k_{1} \geq k_{0}\right)$ s.t. $V\left(A_{k}\right):=V\left(\omega: \tau_{k}(\omega) \leq T\right) \geq \varepsilon$ for all $k \geq k_{1}$. Let $U: R_{n}^{+} \longrightarrow R^{+} \quad$ be $U(x)=\sum_{i=1}^{n}\left(\sqrt{x_{i}}-\right.$ $\left.0.5 \log \left(x_{i}\right)-1\right)$. Set $k \geq k_{0}$ and $T>0$. Using the G-Itô lemma for $\check{V}(t, x)=: U(x)+\int_{t-\tau}^{t}|x(s)|^{2} \mathrm{~d}\langle B\rangle(s), t \in\left[0, \tau_{k} \wedge T\right]$, we get

$$
\begin{aligned}
\mathrm{d} \check{V}(x, t)= & \sum_{i=1}^{n}\left\{\left(0.5 x_{i}^{0.5}-0.5\right)\left(\sum_{j=1}^{n} a_{i j} x_{j}(t-\tau)+b_{i}\right) \mathrm{d}\langle B\rangle(t)+\left(-0.125 x_{i}^{0.5}+0.25\right)\left(\sum_{j=1}^{n} \sigma_{i j} x_{j}\right)^{2} \mathrm{~d}\langle B\rangle(t)\right\} \\
& +\sum_{i=1}^{n}\left(0.5 x_{i}^{0.5}-0.5\right) \sum_{j=1}^{n} \sigma_{i j} x_{j} \mathrm{~d} B(t)+\left(|x|^{2}-|x(t-\tau)|^{2}\right) \mathrm{d}\langle B\rangle(t),
\end{aligned}
$$

and noting that

$$
\sum_{i=1}^{n}\left[\left(0.5 x_{i}^{0.5}-0.5\right)\left(b_{i}+\sum_{j=1}^{n} a_{i j} x_{j}(t-\tau)\right)\right] \leq \frac{1}{2} \sum_{i=1}^{n}\left(x_{i}^{0.5}-1\right) b_{i}+|x(t-\tau)|^{2}+\sum_{i=1}^{n} \sum_{j=1}^{n} \frac{n a_{i j}^{2}}{16}\left(x_{i}^{0.5}-1\right)^{2}
$$

and $\sum_{i=1}^{n}\left(\sum_{j=1}^{n} \sigma_{i j} x_{j}\right)^{2} \leq \sum_{i=1}^{n}|\sigma|^{2} x_{i}^{2}=|\sigma|^{2}|x|^{2}$, as well as $\sum_{i=1}^{n} x_{i}^{0.5}\left(\sum_{j=1}^{n} \sigma_{i j} x_{j}\right)^{2} \geq \sum_{i=1}^{n} \sigma_{i i}^{2} x_{i}^{2.5}$ by the assumption (A).

Thus,

$$
\begin{aligned}
\mathrm{d} \check{V}(t, x(t)) \leq & {\left[\sum_{i=1}^{n} \frac{1}{2}\left(x_{i}^{0.5}-1\right) b_{i}+|x(t)|^{2}+\sum_{i=1}^{n} \sum_{j=1}^{n} \frac{n a_{i j}^{2}}{16}\left(x_{i}^{0.5}-1\right)^{2}\right] \mathrm{d}\langle B\rangle(t)+\left[-0.125 \sum_{i=1}^{n} \sigma_{i i}^{2} x_{i}^{2.5}+0.25|\sigma|^{2}|x|^{2}\right] \mathrm{d}\langle B\rangle(t) } \\
& +\sum_{i=1}^{n}\left(0.5 x_{i}^{0.5}-0.5\right) \sum_{j=1}^{n} \sigma_{i j} x_{j} \mathrm{~d} B(t) .
\end{aligned}
$$


Denote

$$
\begin{aligned}
f(x, a, b, \sigma)= & \sum_{i=1}^{n} \frac{1}{2}\left(x_{i}^{0.5}-1\right) b_{i}+|x|^{2}\left(1+\frac{1}{4}|\sigma|^{2}\right) \\
& +\sum_{i=1}^{n} \sum_{j=1}^{n} \frac{n a_{i j}^{2}}{16}\left(x_{i}^{0.5}-1\right)^{2}-\frac{1}{8} \sum_{i=1}^{n} \sigma_{i i}^{2} x_{i}^{2.5},
\end{aligned}
$$

since we note that there is $K$ s.t. $f(x, a, b, \sigma)$ is bounded, namely, $f(x, a, b, \sigma)<K$, then

$$
\begin{aligned}
\widehat{\mathbb{E}}\left[\int_{\tau_{k} \wedge T-\tau}^{T \wedge \tau_{k}}|x|^{2} \mathrm{~d}\langle B\rangle(s)+U\left(x\left(T \wedge \tau_{k}\right)\right)\right] & \leq \widehat{\mathbb{E}}\left[\int_{-\tau}^{0}|x|^{2} \mathrm{~d}\langle B\rangle(s)\right]+U\left(x_{0}\right)+K \bar{\sigma}^{2} \widehat{\mathbb{E}}\left[T \wedge \tau_{k}\right] \\
& \leq \bar{\sigma}^{2} \int_{-\tau}^{0} \widehat{\mathbb{E}}\left[|x|^{2}\right] \mathrm{d} s+U\left(x_{0}\right)+K \bar{\sigma}^{2} \widehat{\mathbb{E}}\left[T \wedge \tau_{k}\right],
\end{aligned}
$$

then $\widehat{\mathbb{E}}\left[U\left(x\left(\tau_{k} \wedge T\right)\right)\right] \leq U\left(x_{0}\right)+\bar{\sigma}^{2} \int_{-\tau}^{0} \widehat{\mathbb{E}}\left[|x(s)|^{2}\right] \mathrm{d} s+K \bar{\sigma}^{2} T<$ $\infty$. From the definition of $\tau_{k}$, we know $\forall \omega \in A_{k}, \exists$ some $i$ s.t. $x_{i}\left(\tau_{k}, \omega\right) \notin(1 / k, k)$, namely, $x_{i}\left(\tau_{k}\right) \leq 1 / k$, or $x_{i}\left(\tau_{k}\right) \geq k<\infty$. Noting that the function $U\left(x_{i}\right)$ is decreasing when $0<x_{i} \leq 1$ and is increasing when $x_{i}>1$, hence $U\left(x\left(\tau_{k}\right)\right) \geq U(1 / k, \ldots, 1 / k)$ and $U\left(x\left(\tau_{k}\right)\right) \geq U(k, \ldots, \quad k)$, namely, $U\left(x\left(\tau_{k}\right)\right) \geq$ $\max \{\sqrt{(1 / k)}-0.5 \log (1 / k)-1, \sqrt{k}-0.5 \log (k)-1\}$. Therefore, we have

$$
\begin{aligned}
\varepsilon C_{k} \leq C_{k} V\left(A_{k}\right) & \leq \widehat{\mathbb{E}}\left[I_{A_{k}} U\left(x\left(\tau_{k}\right)\right)\right] \leq \widehat{\mathbb{E}}\left[U\left(x\left(T \wedge \tau_{k}\right)\right)\right] \\
& \leq U\left(x_{0}\right)+\bar{\sigma}^{2} \int_{-\tau}^{0} \widehat{\mathbb{E}}\left[|x|^{2}\right] \mathrm{d} t+K \bar{\sigma}^{2} T .
\end{aligned}
$$

Setting $k \longrightarrow \infty$, we have the contradiction $\infty \leq \widehat{\mathbb{E}}\left[U\left(x\left(\tau_{k} \wedge T\right)\right)\right]<\infty$; therefore, we have $\tau_{\infty}=\infty$ q.s., namely, $\tau_{e}=\infty$ q.s., so $v\left(\omega: x(t) \in R_{+}^{n}, t \geq 0\right)=1$.

\section{Asymptotic Behaviors of the Solution}

Theorem 2. Under the assumption $(A)$, if $\widehat{\mathbb{E}}\left[B(1)^{2}\right]=\bar{\sigma}^{2} \leq 1$, for any $\beta \in(0,1)$ and $\delta \in(0,1), \exists C_{0}=C(\delta)>0$ s.t. the solution $x(t)$ of equation (2) is as follows:

$$
\limsup _{t \longrightarrow \infty} V\left(e^{\left(\langle B\rangle(t)-\bar{\sigma}^{2} t\right) / \beta}|x(t)| \leq C_{0}\right) \geq 1-\delta .
$$

Proof. Let

$$
U(x)=\sum_{i=1}^{n} x_{i}^{\beta}
$$

Using the G-Itô lemma for $U(x)$ and noting

$$
\mathrm{d} x_{i}(t)=x_{i}\left[\left(\sum_{j=1}^{n} a_{i j} x_{j}(t-\tau)+b_{i}\right) \mathrm{d}\langle B\rangle(t)+\sum_{j=1}^{n} \sigma_{i j} x_{j} \mathrm{~d} B(t)\right] \text {, }
$$

we have

$$
\begin{aligned}
\mathrm{d} U(x)= & \sum_{i=1}^{n} \beta x_{i}^{\beta}\left[\left(b_{i}+\sum_{j=1}^{n} a_{i j} x_{j}(t-\tau)\right) \mathrm{d}\langle B\rangle(t)+\sum_{j=1}^{n} \sigma_{i j} x_{j} \mathrm{~d} B(t)\right] \\
& +\frac{1}{2} \beta(\beta-1) \sum_{i=1}^{n} x_{i}^{\beta}\left[\sum_{j=1}^{n} \sigma_{i j} x_{j}\right]^{2} \mathrm{~d}\langle B\rangle(t) .
\end{aligned}
$$

Since

$$
\sum_{i=1}^{n} \sum_{j=1}^{n} \beta x_{i}^{\beta} a_{i j} x_{j}(t-\tau) \leq \sum_{i=1}^{n} \sum_{j=1}^{n}\left[\frac{n}{4}\left(\beta a_{i j} x_{i}^{\beta}\right)^{2}+\frac{x_{j}(t-\tau)^{2}}{n}\right] \text {, }
$$

and from equation (13), we get

$$
\mathrm{d} U(x) \leq\left[\sum_{i=1}^{n} \beta b_{i} x_{i}^{\beta}+\frac{n}{4} \sum_{i=1}^{n} \sum_{j=1}^{n}\left(\beta a_{i j} x_{i}^{\beta}\right)^{2}\right] \mathrm{d}\langle B\rangle(t)+\left[\frac{1}{2} \beta(\beta-1) \sum_{i=1}^{n} x_{i}^{\beta+2} \sigma_{i i}^{2}+|x(t-\tau)|^{2}\right] \mathrm{d}\langle B\rangle(t)+\left(\sum_{i=1}^{n} \beta x_{i}^{\beta} \sum_{j=1}^{n} \sigma_{i j} x_{j}\right) \mathrm{d} B(t),
$$


then

$$
\begin{aligned}
d\left(e^{\langle B\rangle(t)} U(x(t))\right)= & e^{\langle B\rangle(t)} U(x(t)) d\langle B\rangle(t)+e^{\langle B\rangle(t)} \mathrm{d} U(x(t)) \\
\leq & e^{\langle B\rangle(t)}\left[\sum_{i=1}^{n}\left(\beta b_{i}+1\right) x_{i}^{\beta}+\frac{n}{4} \sum_{i=1}^{n} \sum_{j=1}^{n}\left(\beta a_{i j} x_{i}^{\beta}\right)^{2}\right] \mathrm{d}\langle B\rangle(t)+\exp (\langle B\rangle(t))\left[|x(t-\tau)|^{2}+0.5 \beta(\beta-1) \sum_{i=1}^{n} x_{i}^{\beta+2} \sigma_{i i}^{2}\right] \mathrm{d}\langle B\rangle(t) \\
& +e^{\langle B\rangle(t)}\left(\sum_{i=1}^{n} \beta x_{i}^{\beta} \sum_{j=1}^{n} \sigma_{i j} x_{j}\right) \mathrm{d} B(t) .
\end{aligned}
$$

We set

$$
\begin{gathered}
F_{1}(x)=\sum_{i=1}^{n}\left(1+\beta b_{i}\right) x_{i}^{\beta}+\frac{n}{4} \sum_{i=1}^{n} \sum_{j=1}^{n}\left(\beta a_{i j} x_{i}^{\beta}\right)^{2} \\
+e^{\tau}|x|^{2}-\frac{1}{2} \beta(1-\beta) \sum_{i=1}^{n} x_{i}^{\beta+2} \sigma_{i i}^{2},
\end{gathered}
$$

then $F_{1}(x)$ is bounded in $R_{+}^{n}$, say $K_{1}$, from (16),

$$
\begin{aligned}
\widehat{\mathbb{E}}\left[e^{\langle B\rangle(t)} U(x(t))\right] & \leq U_{0}+\widehat{\mathbb{E}}\left[\int_{0}^{t} e^{\langle B\rangle(s)}\left(K_{1}-e^{\tau}|x(s)|^{2}+|x(s-\tau)|^{2}\right) \mathrm{d}\langle B\rangle(s)\right] \\
& \leq U_{0}+\widehat{\mathbb{E}}\left[\int_{0}^{t} e^{\bar{\sigma}^{2} s}\left(K_{1}-e^{\tau}|x(s)|^{2}+|x(s-\tau)|^{2}\right) \mathrm{d}\langle B\rangle(s)\right] \\
& \leq U_{0}+\bar{\sigma}^{2} \widehat{\mathbb{E}}\left[\int_{0}^{t} e^{\bar{\sigma}^{2} s}\left(K_{1}-e^{\tau}|x(s)|^{2}+|x(s-\tau)|^{2}\right) \mathrm{d} s\right] \\
& \leq U_{0}+K_{1} e^{\bar{\sigma}^{2} t}+\bar{\sigma}^{2} \widehat{\mathbb{E}}\left[\int_{0}^{t} e^{\bar{\sigma}^{2} s}|x(s-\tau)|^{2}-e^{\bar{\sigma}^{2} s+\tau}|x(s)|^{2} \mathrm{~d} s\right] \\
& =U_{0}+K_{1} e^{\bar{\sigma}^{2} t}+\bar{\sigma}^{2} \widehat{\mathbb{E}}\left[\int_{-\tau}^{t-\tau} e^{\bar{\sigma}^{2}(s+\tau)}|x(s)|^{2}-e^{\bar{\sigma}^{2} s+\tau}|x(s)|^{2} \mathrm{~d} s\right],
\end{aligned}
$$

where $U_{0}=U(x(0))$, and noting that $\bar{\sigma}^{2}$ satisfies $\bar{\sigma}^{2} \leq 1$, by (19), then

$$
\widehat{\mathbb{E}}\left[e^{\langle B\rangle(t)} U(x(t))\right] \leq U_{0}+K_{1} e^{\bar{\sigma}^{2} t}+\bar{\sigma}^{2} \widehat{\mathbb{E}}\left[\int_{-\tau}^{0} e^{\bar{\sigma}^{2}(s+\tau)}|x(s)|^{2} \mathrm{~d} s\right],
$$

therefore,

$$
\limsup _{t \rightarrow \infty} \widehat{\mathbb{E}}\left[e^{\langle B\rangle(t)-\bar{\sigma}^{2} t} U(x(t))\right] \leq K_{1} .
$$

In addition, we note

$$
|x|^{\beta}=\left(\sum_{i=1}^{n} x_{i}^{2}\right)^{\beta / 2} \leq n^{\beta / 2} \max _{1 \leq i \leq n} x_{i}^{\beta} \leq n^{\beta / 2} U(x),
$$

$\forall \delta>0$, let $C_{0}=\left(K_{0} / \delta\right)^{1 / \beta}$, then

$$
\begin{aligned}
v\left(\exp \left(\frac{\langle B\rangle(t)-\bar{\sigma}^{2} t}{\beta}\right)|x|>C_{0}\right) & \leq \frac{\widehat{\mathbb{E}}\left[\exp \left(\langle B\rangle(t)-\bar{\sigma}^{2} t\right)|x|^{\beta}\right]}{C_{0}^{\beta}} \\
& \leq \frac{K_{0}}{C_{0}^{\beta}}=\delta .
\end{aligned}
$$

Hence,

$$
\limsup _{t \longrightarrow \infty} V\left(e^{\left(\langle B\rangle(t)-\bar{\sigma}^{2} t\right) / \beta}|x| \leq C_{0}\right) \geq 1-\delta .
$$

Theorem 3. Suppose the $(A)$ is true, and there exists $K(K>0)$ is independent of $\{x(s): s \in[-\tau, 0]\}$, then 


$$
\underset{T \longrightarrow \infty}{\limsup } \frac{-1}{T} \widehat{\mathbb{E}}\left[\left.\int_{0}^{T} \dashv x\right|^{2} \mathrm{~d}\langle B\rangle(s)\right] \leq K \bar{\sigma}^{2}
$$

Proof. Write (7) as $g(x, a, b, \sigma)=g_{1}(x, a, b, \sigma)-|x|^{2}$, where

$$
\begin{aligned}
g_{1}(x, a, b, \sigma):= & \sum_{i=1}^{n} 0.5\left(x^{0.5}-1\right) b_{i}+\sum_{i=1}^{n} \sum_{j=1}^{n} \frac{n a_{i j}^{2}}{16}\left(x^{0.5}-1\right)^{2} \\
& -0.125 \sum_{i=1}^{n} \sigma_{i i}^{2} x_{i}^{2.5}+|x|^{2}\left(2+0.25|\sigma|^{2}\right)
\end{aligned}
$$

then $g(x, a, b, \sigma) \leq K-|x|^{2}$, where $K:=\max _{x \in R_{+}^{n}} g_{1}(x)<\infty$. Taking expectation from 0 to $\tau_{k} \wedge$ Ton both sides of equation (6), we have

$$
\begin{aligned}
0 \leq \widehat{\mathbb{E}} & {\left[\int_{-\tau}^{0}|x|^{2} \mathrm{~d}\langle B\rangle(s)\right]+U\left(x_{0}\right) } \\
& +\widehat{\mathbb{E}}\left[\int_{0}^{\tau_{k} \wedge T}\left(K-|x|^{2}\right) \mathrm{d}\langle B\rangle(s)\right] \\
\leq & \bar{\sigma}^{2} \int_{-\tau}^{0} \widehat{\mathbb{E}}\left[|x|^{2}\right] \mathrm{d} s+U\left(x_{0}\right)+K \bar{\sigma}^{2} \widehat{\mathbb{E}}\left[T \wedge \tau_{k}\right] \\
& +\widehat{\mathbb{E}}\left[\int_{0}^{T \wedge \tau_{k}}-|x|^{2} \mathrm{~d}\langle B\rangle(s)\right] .
\end{aligned}
$$

Letting $k \longrightarrow \infty$ yields

$-\widehat{\mathbb{E}}\left[\int_{0}^{T}-|x|^{2} \mathrm{~d}\langle B\rangle(s)\right] \leq \bar{\sigma}^{2} \int_{-\tau}^{0} \widehat{\mathbb{E}}\left[|x|^{2}\right] \mathrm{d} s+U\left(x_{0}\right)+K \bar{\sigma}^{2} T$.

Therefore, setting $T \longrightarrow \infty$,

$$
\limsup _{T \rightarrow \infty} \frac{-1}{T} \widehat{\mathbb{E}}\left[\int_{0}^{T}-|x|^{2} \mathrm{~d}\langle B\rangle(s)\right] \leq K \bar{\sigma}^{2} .
$$

\section{Asymptotic Moment Estimations}

Theorem 4. If condition (A) is true, then $\forall\{x(s): s \in[-\tau, 0]\}$, $x(t)$ in (2) satisfies

$$
\limsup _{t \rightarrow \infty} \frac{1}{t} \widehat{\mathbb{E}}\left[\log \left(\frac{|x(t)|}{\sqrt{n}}\right)+\frac{\widehat{\sigma}^{2}}{4 n} \int_{0}^{t}|x(s)|^{2} \mathrm{~d}\langle B\rangle(s)\right] \leq \bar{\sigma}^{2} K,
$$

where $\widehat{\sigma}=\min _{1 \leq i \leq n} \sigma_{i i}$.

Proof. Let $\widetilde{V}(x)=\sum_{i=1}^{n} x_{i}(t)$ for $x \in R_{+}^{n}$, then by G-Itô's lemma of Reference [13], we have

$$
\begin{aligned}
\log (\tilde{V}(x))= & C_{0}+\int_{0}^{t} \frac{x^{T}(s)}{V(x(s))}(b+A x(s-\tau)) \mathrm{d}\langle B\rangle(s) \\
& +\int_{0}^{t} \frac{x^{T}(s) \sigma x(s)}{\tilde{V}(x(s))} \mathrm{d} B(s)-\int_{0}^{t} \frac{\left|x^{T}(s) \sigma x(s)\right|^{2}}{2 \widetilde{V}^{2}(x)} \mathrm{d}\langle B\rangle(s),
\end{aligned}
$$

where $C_{0}=\log (\tilde{\mathrm{V}}(x(0)))$. Noting that

$$
\left\langle\int_{0}^{t} \frac{x^{T} \sigma x}{\widetilde{V}(x)} \mathrm{d} B(s),\left|\int_{0}^{t} \frac{\left|x^{T} \sigma x\right|^{2}}{\widetilde{V}^{2}(x)}\right| \mathrm{d}\langle B\rangle(s)\right\rangle=\int_{0}^{t} \frac{\left|x^{T} \sigma x\right|^{2}}{\widetilde{V}^{2}(x)} \mathrm{d}\langle B\rangle(s),
$$

$\forall \varepsilon \in(0,1 / 2)$, from Lemma 3.1 in reference [19], for any integer $k \geq 1$, we have

$$
V\left(\sup _{0 \leq t \leq k}\left[\int_{0}^{t} \frac{x^{T} \sigma x}{\widetilde{V}(x)} \mathrm{d} B(s)-\frac{\varepsilon}{2} \int_{0}^{t} \frac{\left|x^{T} \sigma x\right|^{2}}{\widetilde{V}^{2}(x)} \mathrm{d}\langle B\rangle(s)\right]>\frac{2}{\varepsilon} \ln k\right) \leq \frac{1}{k^{2}},
$$

so

$$
\sum_{k=1}^{\infty} V\left(\sup _{0 \leq t \leq k}\left[\int_{0}^{t} \frac{x^{T} \sigma x}{\widetilde{V}(x)} \mathrm{d} B(s)-\frac{\varepsilon}{2} \int_{0}^{t} \frac{\left|x^{T} \sigma x\right|^{2}}{\widetilde{V}^{2}(x)} \mathrm{d}\langle B\rangle(s)\right]>\frac{2}{\varepsilon} \ln k\right)<\infty,
$$

applying Lemma 2 in [15], we know for all but finitely many $k$,

$$
\sup _{0 \leq t \leq k}\left[\int_{0}^{t} \frac{x^{T} \sigma x}{\widetilde{V}(x)} \mathrm{d} B(s)-\frac{\varepsilon}{2} \int_{0}^{t} \frac{\left|x^{T} \sigma x\right|^{2}}{\widetilde{V}^{2}(x)} \mathrm{d}\langle B\rangle(s)\right] \leq \frac{\ln k^{2}}{\varepsilon},
$$

quasi-surely true, i.e., $\exists \Omega_{i} \subset \Omega(v(\Omega i)=1)$ s.t. $\forall \omega \in \Omega_{i}$ and $k_{i}=k_{i}(\omega)$ s.t.

$$
\int_{0}^{t} \frac{x^{T}(s) \sigma x}{\widetilde{V}(x)} \mathrm{d} B(s)-\frac{\varepsilon}{2} \int_{0}^{t} \frac{\left|x^{T} \sigma x\right|^{2}}{\widetilde{V}^{2}(x)} d\langle B\rangle(s) \leq \frac{\ln k^{2}}{\varepsilon}, \quad 0 \leq t \leq k,
$$

$k \geq k_{i}(\omega)$. From equation (32) and inequality (37),

$$
\begin{aligned}
\log (\tilde{V}(x)) \leq & C_{0}+\frac{\ln k^{2}}{\varepsilon}+\int_{0}^{t}[\sqrt{n}(|x(s-\tau)|\|A\|+|b|) \\
& \left.-\widehat{\sigma}^{2}|x|^{2} \frac{(1-\varepsilon)}{2 n}\right] \mathrm{d}\langle B\rangle(s),
\end{aligned}
$$

$t \in\left[0, k_{i}(\omega)\right], k \geq k_{i}(\omega)$, in other words,

$$
\begin{aligned}
& \log (\tilde{V}(x))+\widehat{\sigma}^{2} \frac{(1-2 \varepsilon)}{4 n} \int_{0}^{t}|x|^{2} d\langle B\rangle(s) \\
& \quad \leq C_{0}+\frac{\ln k^{2}}{\varepsilon}+\int_{0}^{t}\left[\sqrt{n}(|x(s-\tau)|\|A\|+|b|)-|x|^{2} \frac{\widehat{\sigma}^{2}}{4 n}\right] d\langle B\rangle(s),
\end{aligned}
$$

where $\widehat{\sigma}=\min \sigma_{i i}(i \in[1, n])$. Taking $G$-expectation $\widehat{\mathbb{E}}$ for (39), and then $\forall \omega \in \cap_{i=1}^{n} \Omega_{i}$, from (39), we get 


$$
\begin{aligned}
\widehat{\mathbb{E}}[ & \left.\widehat{\sigma}^{2} \frac{(1-2 \varepsilon)}{4 n} \int_{0}^{t}|x|^{2} \mathrm{~d}\langle B\rangle(s)+\log (\widetilde{V}(x))\right] \\
\leq & \frac{\ln k^{2}}{\varepsilon}+\bar{\sigma}^{2} \widehat{\mathbb{E}}\left[\int_{0}^{t}(|x(s-\tau)|\|A\|+|b|) \sqrt{n}-\frac{\widehat{\sigma}^{2}}{4 n}|x|^{2} \mathrm{~d} s\right]+C_{0} \\
\leq & C_{0}+\frac{\ln k^{2}}{\varepsilon}+\sqrt{n}\|A\| \bar{\sigma}^{2} \widehat{\mathbb{E}}\left[\int_{-\tau}^{0}|x| \mathrm{d} s\right] \\
& +\bar{\sigma}^{2} \widehat{\mathbb{E}}\left[\int_{0}^{t} \sqrt{n}(|b|+\|A\||x|)-\frac{\widehat{\sigma}^{2}}{4 n}|x|^{2} \mathrm{~d} s\right] \\
\leq & C_{0}+\frac{2 \ln k}{\varepsilon}+\sqrt{n}\|A\| \bar{\sigma}^{2} \widehat{\mathbb{E}}\left[\int_{-\tau}^{0}|x| \mathrm{d} s\right]+\bar{\sigma}^{2} K t,
\end{aligned}
$$

where $\sqrt{n}(\|A\||x|+|b|)-\left(\widehat{\sigma}^{2} / 4 n\right)|x(s)|^{2} \leq K$. Set $\max \left\{k_{i}(\omega)\right.$, $i \in[1, n]\}=k_{0}(\omega)$, then $\forall \omega \in \cap_{i=1}^{n} \Omega_{i}, t \in[k-1, k], k \geq k_{0}(\omega)$, it gets from (40):

$\limsup _{t \rightarrow \infty} \frac{1}{t} \widehat{\mathbb{E}}\left[\log (\widetilde{V}(x))+\frac{\widehat{\sigma}^{2}(1-2 \varepsilon)}{4 n} \int_{0}^{t}|x|^{2} d\langle B\rangle(s)\right] \leq \bar{\sigma}^{2} K$.

Letting $\varepsilon$ tend to zero and noting that $|x| \leq \sqrt{n} V(x)$ yield $\limsup _{t \rightarrow \infty} \frac{1}{t} \widehat{\mathbb{E}}\left[\log \left(\frac{|x(t)|}{\sqrt{n}}\right)+\frac{\widehat{\sigma}^{2}}{4 n} \int_{0}^{t}|x(s)|^{2} d\langle B\rangle(s)\right] \leq \bar{\sigma}^{2} K$.

The proof is complete.

\section{Data Availability}

No data were used to support this study.

\section{Conflicts of Interest}

The authors declare that they have no conflicts of interest to this work.

\section{Acknowledgments}

This research was supported by the NSF-China (nos. 11761028 and 11501009), NSF-Anhui Province (1508085 JGD10), HTIT and RTFS-Yunnan Province, and Honghe University (nos. 2015HB061, 2014HB0204, and 2018JS480).

\section{References}

[1] A. J. Lotka, Elements of Physical Biology, William and Wilkins, Baltimore, MD, USA, 1925.

[2] V. Volterra, "Variazioni e fluttuazioni del numero dindividui in specie d'animani conviventi," Mem. Acad. Lincei, vol. 2, pp. 31-113, 1926.

[3] Y. Lou, S. Martínez, and P. Poláčik, "Loops and branches of coexistence states in a Lotka-Volterra competition model," Journal of Differential Equations, vol. 230, no. 2, pp. 720-742, 2006.

[4] Y. Zhang and M. Wang, "Bifurcation of Lotka-Volterra competition model with nonlinear boundary conditions," Applied Mathematics Letters, vol. 38, pp. 52-56, 2014.
[5] X. Mao, G. Marion, and E. Renshaw, "Environmental Brownian noise suppresses explosions in population dynamics," Stochastic Processes and Their Applications, vol. 97, no. 1, pp. 95-110, 2002.

[6] X. Mao, S. Sabanis, and E. Renshaw, "Asymptotic behaviour of the stochastic Lotka-Volterra model," Journal of Mathematical Analysis and Applications, vol. 287, no. 1, pp. 141-156, 2003.

[7] A. Hastings, "Global stability in Lotka-Volterra systems with diffusion," Journal of Mathematical Biology, vol. 6, no. 2, pp. 163-168, 1978.

[8] N. H. Du and V. H. Sam, "Dynamics of a stochastic LotkaVolterra model perturbed by white noise," Journal of Mathematical Analysis and Applications, vol. 324, no. 1, pp. 82-97, 2006.

[9] A. Bahar and X. Mao, "Stochastic delay Lotka-Volterra model," Journal of Mathematical Analysis and Applications, vol. 292, no. 2, pp. 364-380, 2004.

[10] F. Wu and Y. Xu, "Stochastic Lotka-Volterra population dynamics with infinite delay," SIAM Journal on Applied Mathematics, vol. 70, no. 3, pp. 641-657, 2009.

[11] Y. Huang, Q. Liu, and Y. Liu, "Global asymptotic stability of a general stochastic Lotka-Volterra system with delays," Applied Mathematics Letters, vol. 26, no. 1, pp. 175-178, 2013.

[12] S. Peng, "G-expectation, $G$-brownian motion and related stochastic calculus of Itô type," in Stochastic Analysis and Applications, Abel Symp. 2, F. E. Benth, G. Di Nunno, T. Lindstrøm et al., Eds., pp. 541-567, Springer, Berlin, Germany, 2007.

[13] S. Peng, "Nonlinear expectations and stochastic calculus under uncertainty-with robust central limit theorem and $G$ Brownian motion," 2010, https://arxiv.org/abs/1002.4546.

[14] S. Peng, "Backward stochastic differential equation, nonlinear expectation and their applications," in Proceedings of the International Congress of Mathematicians, World Scientific, Singapore, pp. 393-432, 2010.

[15] Z. Chen, "Strong laws of large numbers for sub-linear expectations," Science China Mathematics, vol. 59, no. 5, pp. 945-954, 2016.

[16] L. Denis, M. Hu, and S. Peng, "Function spaces and capacity related to a sublinear expectation: application to G-brownian motion paths," Potential Analysis, vol. 34, no. 2, pp. 139-161, 2011.

[17] H. M. Soner, N. Touzi, and J. Zhang, "Martingale representation theorem for the G-expectation," Stochastic Processes and Their Applications, vol. 121, no. 2, pp. 265-287, 2011.

[18] F. Gao, "Pathwise properties and homeomorphic flows for stochastic differential equations driven by G-brownian motion," Stochastic Processes and Their Applications, vol. 119, pp. 3356-3382, 2009.

[19] D. Zhang and Z. Chen, "Exponential stability for stochastic differential equation driven by G-brownian motion," Applied Mathematics Letters, vol. 25, no. 11, pp. 1906-1910, 2012.

[20] W. Yu, Y. Yong, G. Guan, Y. Huang, W. Su, and C. Cui, "Valuing guaranteed minimum death benefits by cosine series expansion," Mathematics, vol. 7, no. 9, p. 835, 2019.

[21] Z. Zhang, Y. Yong, and W. Yu, "Valuing equity-linked death benefits in general exponential Lévy models," Journal of Computational and Applied Mathematics, vol. 365, Article ID 112377, 2020.

[22] W. Yu, F. Wang, Y. Huang, and H. Liu, "Social Optimal mean field control problem for population growth model," Asian Journal of Control, vol. 21, 2019. 